\title{
Multi-jet merging for vector boson plus jets including electroweak corrections
}

\section{S. Kallweit}

Institut für Physik \& PRISMA Cluster of Excellence, Johannes Gutenberg Universität, 55099 Mainz, Germany

E-mail: kallweiteuni-mainz.de

\section{J. M. Lindert}

Physik-Institut, Universität Zürich, Winterthurerstrasse 190, CH-8057 Zürich, Switzerland

E-mail: lindertephysik.uzh.ch

\section{P. Maierhöfer*}

Physikalisches Institut, Albert-Ludwigs-Universität Freiburg, 79104 Freiburg, Germany

E-mail: philipp.maierhoefer@physik.uni-freiburg.de

\section{S. Pozzorini}

Physik-Institut, Universität Zürich, Winterthurerstrasse 190, CH-8057 Zürich, Switzerland

E-mail: pozzorin@physik.uzh.ch

\section{Schönherr}

Physik-Institut, Universität Zürich, Winterthurerstrasse 190, CH-8057 Zürich, Switzerland

E-mail: marek.schoenherrephysik.uzh.ch

\begin{abstract}
We report on next-to-leading order (NLO) QCD and electroweak (EW) corrections to off-shell vector boson production in association with up to two jets. Fixed-order NLO QCD+EW inclusive predictions for vector boson plus jet production are known to exhibit huge QCD corrections and a pathological behaviour of the EW corrections. This problem is solved by applying multi-jet merging with an additional jet at NLO. To this end we employ an approximate method to include NLO EW corrections in the MEPs@NLO multi-jet merging approach. The simulations are performed with the SHERPA and MUNICH Monte Carlo frameworks using NLO QCD and EW matrix elements from OPENLOOPS.
\end{abstract}

Loops and Legs in Quantum Field Theory

24-29 April 2016

Leipzig, Germany

\footnotetext{
* Speaker.
} 


\section{Introduction}

The production of electroweak vector bosons in association with jets is of prime importance in tests of the Standard Model and as a background in Beyond Standard Model physics searches. Particularly for the latter, the inclusion of electroweak corrections is crucial due to large effects arising from EW Sudakov logarithms in the $\mathrm{TeV}$ region.

First NLO EW predictions for vector-boson production in association with more than one jet have been presented for $p p \rightarrow \ell^{+} \ell^{-} j j$ [1] and for on-shell $\mathrm{W}^{+}$-boson production with up to three associated jets at NLO QCD+EW [2]. In the following, we review NLO QCD+EW results for $p p \rightarrow V+$ jets merged up to two jets and with off-shell vector-boson decays into leptons and neutrinos in the complex-mass scheme [3]. I.e. we perform full $2 \rightarrow 3$ and $2 \rightarrow 4$ calculations with NLO QCD and NLO EW corrections. While in this report we limit ourselves to the case of $p p \rightarrow \ell^{-} \bar{v}_{\ell}+1,2$ jets, the complete results with all possible leptonic signatures and a wider range of observables can be found in [4].

The multi-jet merging solves the problems of huge QCD $k$ factors and pathological EW corrections which plagues inclusive fixed order $V+1$ jet production [5]. The inclusion of NLO EW corrections is achieved by combining virtual contributions with an appropriate infra-red subtraction in the MEPs@NLO multi-jet merging framework [6, 7]. This procedure guarantees parton-shower resummation and NLO accuracy in all phase-space regions with up to 2 jets in our simulation. Our predictions are obtained within the NLO QCD+EW framework [2] which uses the OPENLOOPS [8] 1-loop matrix element generator in combination with the MUNICH [9] and SHERPA [10] Monte Carlo programs.

\section{NLO QCD+EW automation}

The calculation of NLO QCD corrections to SM processes has been automated in the OPENLOOPS +SHERPA/MUNICH frameworks and is already well established. More recently, these tools have been upgraded to handle EW corrections in an automated way [2]. The OpEnLOOPS matrix element generator is based on a numerical recursion for the generation of one-loop scattering amplitudes [11]. Tree and loop amplitudes are built from generic building blocks corresponding to the Feynman rules of the EW Standard Model, UV counterterms [12] and rational terms of type $R_{2}$ [13]. Combined with the COLLIER tensor reduction library [14] the employed recursion achieves very high CPU performance and a high degree of numerical stability. The two independent Monte Carlo generators MUNICH and SHERPA handle the bookkeeping of partonic subprocesses, phase-space integration and the subtraction of QCD and QED bremsstrahlung in a way that allows us to consistently include complete $\alpha_{S}^{n} \alpha^{m}$ orders with all QCD-EW interference effects.

\section{Fixed order $V+$ jets production}

We study the production and decay of electroweak bosons in association with one and two jets at NLO QCD+EW, including off-shell effects. The leading order (LO) contribution of $\mathscr{O}\left(\alpha_{\mathrm{S}} \alpha^{2}\right)$ of $V+1$ jet receives NLO QCD corrections of $\mathscr{O}\left(\alpha_{S}^{2} \alpha^{2}\right)$ and NLO EW corrections of $\mathscr{O}\left(\alpha_{\mathrm{S}} \alpha^{3}\right)$. In the $V+2$ jets case, the EW corrections of $\mathscr{O}\left(\alpha_{S}^{2} \alpha^{3}\right)$ can also be regarded as QCD corrections to the 
"LO mix" contribution of $\mathscr{O}\left(\alpha_{\mathrm{S}} \alpha^{3}\right)$ which is included in the simulations as well. The sub-leading Born contribution of $\mathscr{O}\left(\alpha^{4}\right)$, which is dominated by diboson production with semi-leptonic decays, is omitted in order to avoid a double counting between diboson and $V+$ jets processes. Similarly, sub-leading NLO contributions of $\mathscr{O}\left(\alpha_{\mathrm{S}} \alpha^{4}\right)$ or $\mathscr{O}\left(\alpha^{5}\right)$ are not considered.

We employ two different prescriptions to combine QCD and EW NLO corrections, namely additive,

$$
\sigma_{\mathrm{QCD}+\mathrm{EW}}^{\mathrm{NLO}}=\sigma^{\mathrm{LO}}\left(1+\delta \sigma_{\mathrm{QCD}}^{\mathrm{NLO}}+\delta \sigma_{\mathrm{EW}}^{\mathrm{NLO}}\right)
$$

and multiplicative,

$$
\sigma_{\mathrm{QCD} \times \mathrm{EW}}^{\mathrm{NLO}}=\sigma^{\mathrm{LO}}\left(1+\delta \sigma_{\mathrm{QCD}}^{\mathrm{NLO}}\right)\left(1+\delta \sigma_{\mathrm{EW}}^{\mathrm{NLO}}\right)
$$

The difference between the two prescriptions can be regarded as an estimate of unknown higherorder corrections of QCD-EW mixed type. The applied cuts in the simulations correspond to the ATLAS analysis of [15].

It is well known that NLO QCD predictions for $V+1$ jet production suffer from a very poor convergence of the perturbative expansion [5] at large jet transverse momenta. In this kinematic regime the NLO QCD cross section is dominated by dijet configurations where the hardest jet recoils against a similarly hard second jet, while the vector boson remains relatively soft. Such configurations are effectively described at LO, with correspondingly large scale uncertainties. This effect clearly shows in Fig. 1 (left), where the NLO QCD and EW effects in $p p \rightarrow \ell^{-} \bar{v}_{\ell}+1$ jet are plotted versus the transverse momenta of the reconstructed vector boson and of the leading jet.

In the W-boson $p_{\mathrm{T}}$ distribution, QCD corrections are at the level of 40-50\%, but reach $100 \%$ in tail. NLO EW corrections present a consistent Sudakov shape, with corrections growing negative and reaching a few tens of percent in the tail. However, as reflected in the sizeable disparity between additive $\mathrm{QCD}+\mathrm{EW}$ and multiplicative $\mathrm{QCD} \times \mathrm{EW}$ combinations, the large size of NLO QCD and NLO EW effects suggests the presence of important uncontrolled mixed NNLO QCD-EW corrections.

QCD corrections in the jet- $p_{\mathrm{T}}$ distribution show a clear pathological behaviour with several $100 \%$ corrections in the multi-TeV region. Furthermore we observe a pathological NLO EW behaviour, with large positive corrections instead of negative Sudakov effects. In principle, this behaviour can be avoided by imposing a cut that renders the $V+1$ jet cross section sufficiently exclusive with respect to extra jet radiation. As shown in Fig. 1 (centre), a veto against dijet configurations with angular separation $\Delta \phi_{j_{1} j_{2}}>3 \pi / 4$ leads to well-behaved QCD predictions and a standard NLO EW Sudakov behaviour.

However, in order to obtain a precise theoretical description of inclusive $V+$ jets production at high $p_{\mathrm{T}}$, it is clear that fixed-order NLO QCD+EW calculations for one-jet final states have to be supplemented by corresponding predictions for multi-jet final states. As shown in Fig. 1 (right) for the $p_{\mathrm{T}}$ distribution of the hardest jet in $p p \rightarrow V+2$ jets, in this process NLO QCD+EW effects are completely free from the perturbative instabilities that plague NLO predictions for $V+1$ jet production. 

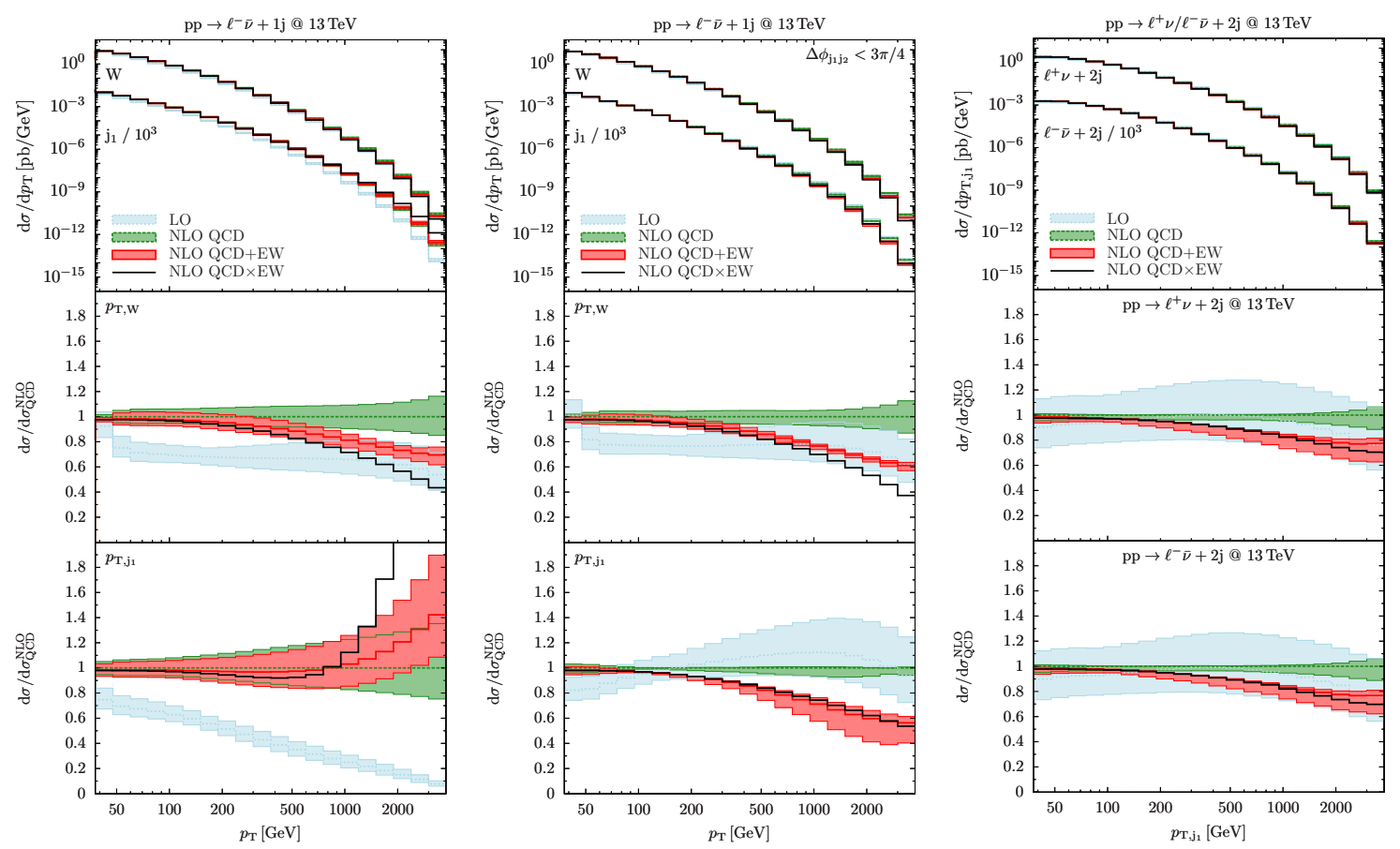

Figure 1: Distributions in the transverse momenta of the reconstructed vector boson, $p_{\mathrm{T}, \mathrm{V}}$, and of the hardest jet, $p_{\mathrm{T}, j_{1}}$, for $p p \rightarrow \ell^{-} \bar{v}_{\ell}+1$ jet with standard cuts (left) and with an additional cut $\Delta \phi_{j_{1} j_{2}}<3 \pi / 4$ (centre), and distributions of the hardest jet for $p p \rightarrow \ell^{-} \bar{v}_{\ell} / \ell^{+} v_{\ell}+2$ jets (right) at $13 \mathrm{TeV}$. Absolute LO (light blue), NLO QCD (green), NLO QCD+EW (red) and NLO QCD $\times$ EW (black) predictions (upper panel) and relative corrections with respect to NLO QCD (lower panels). The bands correspond to scale variations, and in the case of ratios only the numerator is varied. The absolute predictions in $p_{\mathrm{T}, j_{1}}$ are rescaled by a factor $10^{-3}$.

\section{V+jets multi-jet merging at NLO QCD+EW}

For the multi-jet merging we employ the MEPs@NLO method [6, 7] which upgrades LO merging to NLO QCD. However, the technical ingredients for NLO QCD+EW accurate merging are still missing to date. The fact that EW Sudakov logarithms, which are the main source of large NLO EW effects at high energy, arise only from virtual corrections, motivates us to include EW effects in the multi-jet merging by supplementing virtual EW corrections with an appropriate infra-red subtraction without requiring to integrate real-emission matrix elements. We adopt the following $\mathrm{EW}_{\text {virt }}$ approximation for the NLO EW corrections to $V+n$ jet production,

$$
\mathrm{d} \sigma_{n, \mathrm{NLOEW}_{\text {virt }}}=\left[\mathrm{B}_{n}\left(\Phi_{n}\right)+\mathrm{V}_{n, \mathrm{EW}}\left(\Phi_{n}\right)+\mathrm{I}_{n, \mathrm{EW}}\left(\Phi_{n}\right)\right] \mathrm{d} \Phi_{n} .
$$

Here, $\mathrm{B}_{n}\left(\Phi_{n}\right)$ stands for the Born contribution of $\mathscr{O}\left(\alpha_{\mathrm{S}}^{n} \alpha^{2}\right)$, and $\mathrm{V}_{n \text {,EW }}\left(\Phi_{n}\right)$ denotes the exact oneloop EW corrections of $\mathscr{O}\left(\alpha_{\mathrm{S}}^{n} \alpha^{3}\right)$. The cancellation of virtual infra-red singularities is implemented through the $\mathrm{I}_{n, \mathrm{EW}}\left(\Phi_{n}\right)$ term, which represents the NLO EW generalisation of the Catani-Seymour I operator [16, 17]. This corresponds to an approximate and fully inclusive description of the emission of photons and QCD partons at $\mathscr{O}\left(\alpha_{S}^{n} \alpha^{3}\right)$. It turns out that the virtual EW approximation can provide percent-level accuracy for a wide range of observables and energy scales, also well 

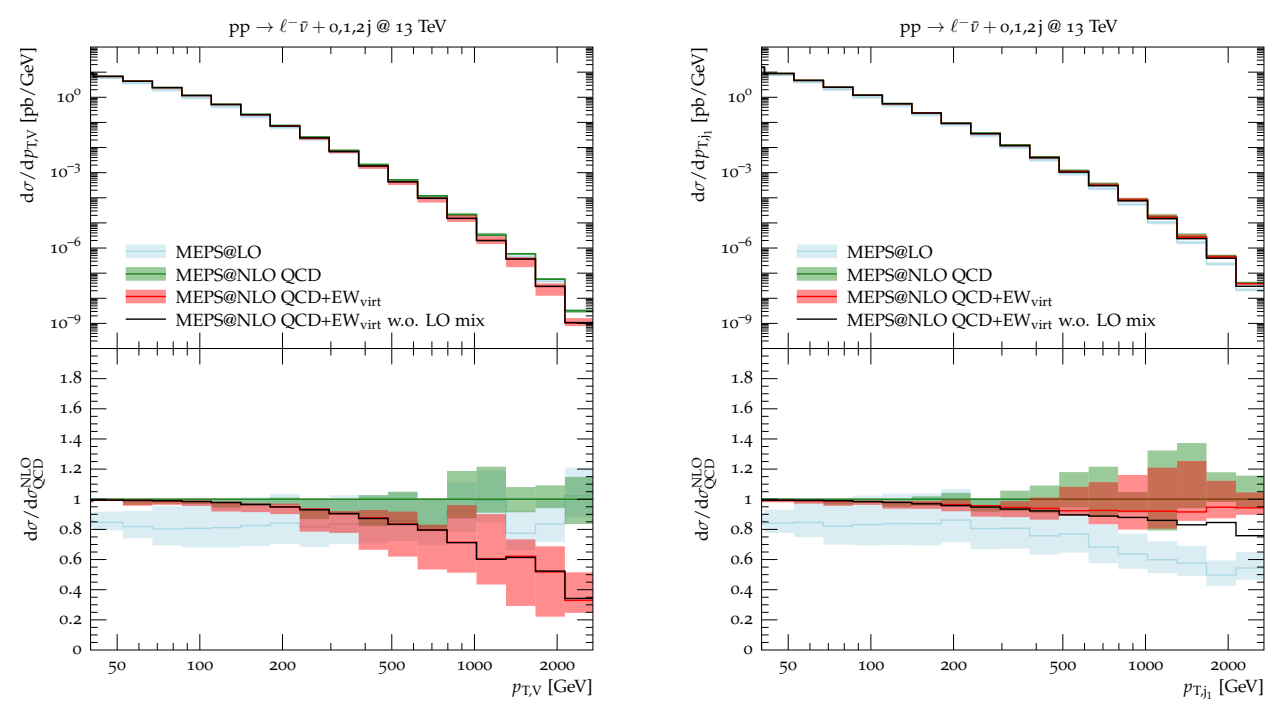

Figure 2: Distributions in the transverse momentum of the reconstructed vector boson (left) and of the hardest jet (right) in $\ell^{-} \bar{v}_{\ell}+$ jets production. The upper frame displays absolute predictions obtained with MEPs@NLO QCD merging (green) and its extension to NLO QCD+EW accuracy including (red) or excluding (black) mixed Born contributions to $V+2$ jet topologies (LO mix). Relative corrections with respect to MEPs@NLO QCD are shown in the lower panels. The bands correspond to scale variations, and in the case of ratios only the numerator is varied.

beyond the kinematic regions where Sudakov EW logarithms become large. The most striking exception is given by the $m_{\ell \ell}$ and $m_{\ell v}$ invariant-mass distributions in the off-shell region below the Breit-Wigner peak. In this case, real QED radiation off the charged leptons leads to corrections of a few tens of percent, which can not be reproduced by the $\mathrm{EW}_{\text {virt }}$ approximation as exclusive real photon emission is not included. In contrast, for distributions in the transverse momentum of the vector bosons or of the charged leptons that arise from their decays, we observe very good agreement, typically at the $1-2 \%$ level, from low $p_{\mathrm{T}}$ up to the multi-TeV region [4].

Fig. 2 (left) shows the transverse momentum of the reconstructed $\mathrm{W}$ boson in $\ell^{-} \bar{v}_{\ell}+$ jets production. This observable receives significant contributions from two-jet topologies, which are, however, typically dominated by a first hard jet, while the second jet tends to be much softer. At high $p_{\mathrm{T}}$ the impact of EW effects in the MEPs framework turns out to be large and can reach $-50 \%$ or more in the multi-TeV region. This is quantitatively consistent with the outcome of the factorised $\mathrm{QCD} \times \mathrm{EW}$ prescription in inclusive $V+$ jet NLO calculations, and clearly more pronounced than what results from the additive combination of QCD+EW fixed-order corrections (Fig. 1). This can be attributed to the inclusion of NLO EW effects in two-jet topologies and, to some extent, also in three-jet topologies via NLO matching to the parton shower. For the vector-boson $p_{\mathrm{T}}$ distribution, mixed Born contributions are almost negligible.

In Fig. 2 (right) we examine the transverse momentum of the leading jet. As this observable exhibits a strong sensitivity to higher jet multiplicities, in particular to topologies with two hard back-to-back jets, it is ideally suited to be calculated using a consistent multi-jet merging. Thanks to the inclusion of dijet topologies as genuine $\ell^{-} \bar{v}_{\ell}+2$ jet production processes at NLO, 
the MEPs@NLO method allows one to avoid giant $K$-factors and cures the pathological behaviour of EW corrections observed in fixed-order NLO QCD+EW calculations for $V+1$ jet. It is worth noting that there is a sizeable impact of mixed Born contributions in the tail of this distribution.

\section{Conclusions}

The Sherpa+OpenLoops and Munich+OpenLoops Monte Carlo frameworks have recently been upgraded to include NLO EW corrections in simulations for in principle arbitrary Standard Model processes in an automated way. Electroweak Sudakov logarithms yield corrections of the order of several $10 \%$ in the $\mathrm{TeV}$ range in distributions of kinematic quantities and tend to largely exceed the uncertainties of NLO QCD predictions.

We developed an approximate framework for multi-jet merging which includes QCD and EW corrections by combining exact EW virtual corrections with an inclusive treatment of photon real radiation. This approach is justified by the fact that the dominating source of EW corrections, namely the Sudakov logarithms, arises only from virtual corrections. The agreement with respect to the full calculation is at the few percent level in the regime where these logarithms become large. With the described procedure, we added NLO EW corrections to the MEPs@ NLO multi-jet merging framework of SHERPA.

Within this MEPs@NLO QCD+EW framework we have provided multi-jet-merged predictions for $V+$ jets production up to two jets including off-shell leptonic decays. The inclusion of dijet topologies as genuine $V+2$ jet production processes at NLO stabilises the perturbative convergence and cures the pathological behaviour of EW corrections observed in fixed-order NLO QCD+EW calculations for $V+1$ jet.

\section{References}

[1] A. Denner, L. Hofer, A. Scharf and S. Uccirati, Electroweak corrections to lepton pair production in association with two hard jets at the LHC, 1411.0916.

[2] S. Kallweit, J. M. Lindert, P. Maierhöfer, S. Pozzorini and M. Schönherr, NLO electroweak automation and precise predictions for W+multijet production at the LHC, JHEP 04 (2015) 012, [1412.5157].

[3] A. Denner et al., Electroweak corrections to charged-current $e^{+} e^{-} \rightarrow 4$ fermion processes: Technical detail results, Nucl.Phys. B724 (2005) 247-294, [hep-ph/ 0505042$].$

[4] S. Kallweit, J. M. Lindert, P. Maierhöfer, S. Pozzorini and M. Schönherr, $N L O Q C D+E W$ predictions for $V+$ jets including off-shell vector-boson decays and multijet merging, JHEP 04 (2016) 021, [1511.08692].

[5] M. Rubin, G. P. Salam and S. Sapeta, Giant QCD K-factors beyond NLO, JHEP 09 (2010) 084, [1 006.2144$]$.

[6] S. Höche, F. Krauss, M. Schönherr and F. Siegert, QCD matrix elements + parton showers: The NLO case, JHEP 1304 (2013) 027, [1207.5030]. 
[7] T. Gehrmann, S. Höche, F. Krauss, M. Schönherr and F. Siegert, NLO QCD matrix elements + parton showers in $e^{+} e^{-} \longrightarrow$ hadrons, JHEP 01 (2013) 144, [1207.5031].

[8] F. Cascioli, J. M. Lindert, P. Maierhöfer and S. Pozzorini, The OpenLoops one-loop generator, https: / /openloops.hepforge.org.

[9] S. Kallweit, MUNICH: “Multi-channel integrator at Swiss (ch) precision”-an automated parton level $N L O$ generator. In preparation.

[10] T. Gleisberg et al., Event generation with SHERPA 1.1, JHEP 0902 (2009) 007, [0811.4622].

[11] F. Cascioli, P. Maierhöfer and S. Pozzorini, Scattering Amplitudes with Open Loops, Phys.Rev.Lett. 108 (2012) 111601, [1111.5206].

[12] A. Denner, Techniques for calculation of electroweak radiative corrections at the one loop level and results for W physics at LEP-200, Fortsch.Phys. 41 (1993) 307-420, [0709.1075].

[13] M. Garzelli, I. Malamos and R. Pittau, Feynman rules for the rational part of the Electroweak 1-loop amplitudes, JHEP 1001 (2010) 040, [0 910 . 3130].

[14] A. Denner, S. Dittmaier and L. Hofer, Collier: a fortran-based Complex One-Loop LIbrary in Extended Regularizations, 1604.06792.

[15] ATLAS collaboration, G. Aad et al., A measurement of the ratio of the production cross sections for $W$ and $Z$ bosons in association with jets with the ATLAS detector, Eur. Phys. J. C74 (2014) 3168, [1408.6510].

[16] S. Dittmaier, A General approach to photon radiation off fermions, Nucl.Phys. B565 (2000) 69-122, [hep-ph/9904440].

[17] S. Dittmaier, A. Kabelschacht and T. Kasprzik, Polarized QED splittings of massive fermions and dipole subtraction for non-collinear-safe observables, Nucl.Phys. B800 (2008) 146-189, [0802.1405]. 